\title{
PERIPHERAL TUBERCULOUS LYMPHADENITIS IN CHILDHOOD
}

\author{
By F. J. W. MilleR, M.D., F.R.C.P., D.C.H. \\ Reader in Social Paediatrics, Department of Child Health, University of Durham; Physician, Children's Department, \\ Royal Victoria Infirmary, Newcastle upon Tyne
}

In this article I shall set out as shortly and clearly as I can the present position regarding the origin and treatment of peripheral tuberculous lymphadenitis in childhood. The title is limited and precise; peripheral lymphadenitis includes all 'superficial ' tuberculous lymphadenitis; mediastinal and mesenteric lymphadenitis are excluded because they have a different natural history. The statement is limited to childhood because I suspect that both the origin and course of tuberculous lymphadenitis may be different in adult life.

The data given refer to the condition as I have seen it in the North of England, the epidemiology and possibly the natural history may be different in other countries but the principles of treatment are likely to be the same.

\section{Epidemiology}

In the British Isles the number of children seen with tuberculous lymphadenitis is steadily declining; this decline is particularly noticeable in children recently infected and presenting with primary tuberculosis with visibly enlarged cervical or other superficial glands. Children infected years ago are still coming forward as their glands undergo belated softening.

In the past there has been a tendency to equate superficial tuberculous lymphadenitis with bovine infection and, in general, this was true of tonsillar adenitis with the primary focus in a tonsil or in the oropharynx. These children sometimes later showed evidence of tuberculous mesenteric adenitis in addition.

The tonsillar gland is indeed by far the commonest site of tuberculous lymphadenitis, but tuberculous infections of the submandibular, preand post-auricular or occipital axillary or inguinal groups of glands are seen and can sometimes be related to visible primary foci in the lymphatic drainage areas (Fig. I ; Miller and Cashman, 1955). When infection is recent and the primary focus is found there is often a history of trauma to the skin or mucous membrane (Miller, I953). It is also important to realize that in infants and young children contact with an infective adult may be of followed by tonsillar cervical adenitis and here the $\overrightarrow{0}$ organism is human in type. The following case history of twins with a limited short exposure makes this point.

\section{Twins with Identical Exposure \\ One Tonsillar Primary Infection and One Pulmonary Infection}

Twin boys were seen when eight months old. The first children in the family, both were well at birth an 0 remained so for four months. During the first s weeks of their life an uncle, the mother's brother, had lived in the house and two months after leaving he hat been found to have active pulmonary tuberculosis.

The first signs of tuberculosis in the twins appeared $\overline{\bar{O}}$ when one of them developed a painless swelling in the right tonsillar gland which slowly enlarged until he was seen at eight months. On examination he had bilateral 2 cervical adenitis, greater on the right than left, and $\overrightarrow{\vec{O}}$ enlarged tonsils with an irregular ulcer visible on the $\frac{0}{3}$ surface of the right tonsil; his spleen was enlarged and $\vec{D}$ his skin tuberculin test was positive. He was given streptomycin and I.N.H., and his tonsils and the enlarged glands on the right side were removed. Histo- $\overline{0}$ logical examination of the tonsils showed tuberculous disease present in the right side but not the left and the right tonsillar gland was caseous.

The other child was also tuberculin positive and an $\mathrm{X}$-ray film showed an active primary focus in the lower lobe of the right lung which later became collapsed but expanded when granulation tissue was sucked out $O$ through a bronchoscope. He had no other signs of tuberculosis. Both twins were treated with chemo- $\frac{D}{O}$ therapy for 18 months and have not developed any further signs of tuberculosis.

In most cities and over increasingly large areas of the country milk must now either be heat- N treated before distribution or come from Tubercu- $\sigma$ lin Tested herds. The position at the end of 19570 is shown in Fig. 2, and at that time 66 per cent. of $\frac{\mathscr{D}}{\mathscr{D}}$

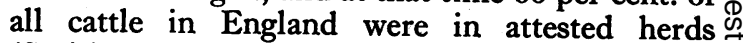
(Smith, 1958).

The number of infective adults in the population 0 is also steadily declining and there is now no $\stackrel{\mathbb{D}}{\mathrm{D}}$ interval between diagnosis and the institution of $\frac{\mathbb{\Phi}}{\otimes}$ 


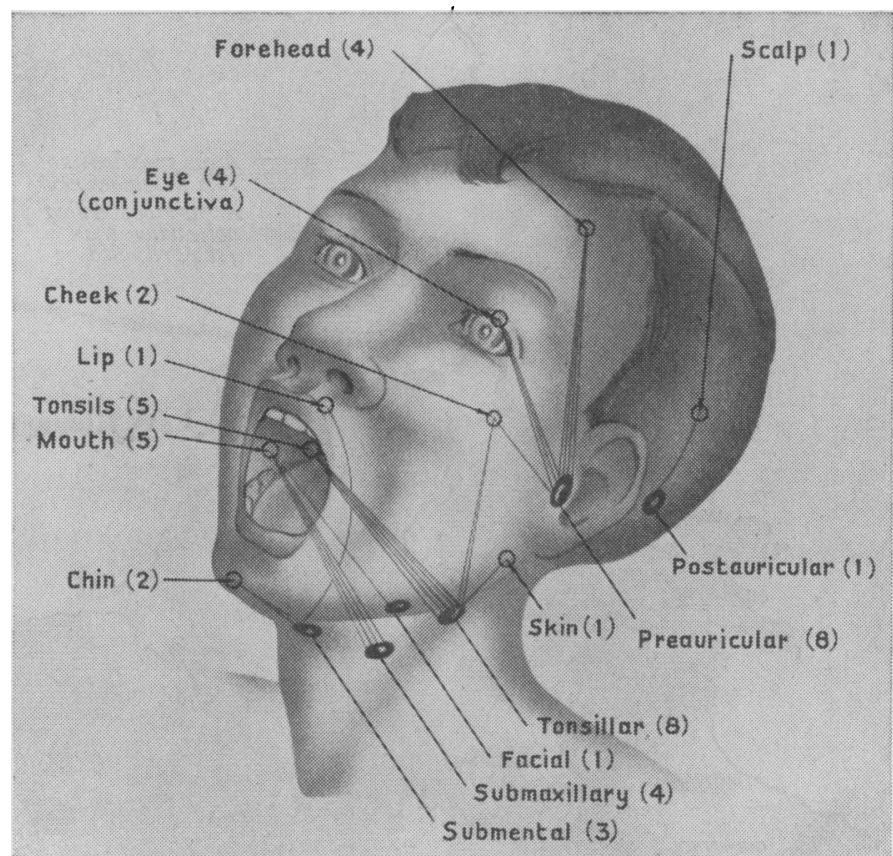

FIG. I.-Relationship between site of primary infection and regional glands first infected in head and neck (Miller and Cashman, r955). (Reproduced by kind permission of 'The Lancet').

effective treatment, so the chance of any child getting a primary tuberculous infection from either a human or bovine source is becoming progressively less.

There are still, however, two dangerous situations: the young infant in contact with an infective parent or other adult before the active pulmonary disease is detected, and the local outbreak of milk-borne infection from an unsuspected bovine source such as occurred in the following history.

\section{Local Outbreak of Cervical Adenitis from Infected Milk}

In the last week of June 1957 a boy, aged four years, and his mother had an attack of acute tonsillitis. The mother made a quick recovery but the child remained pale and listless and in a few days developed attacks of abdominal colic which culminated in vomiting. When first seen Io days after the sore throat he was febrile with some generalized abdominal distension, bowel sounds were present, but there was a resistance to palpation on the right side of the abdomen with the suggestion of an underlying mass. The diagnosis was uncertain and he was admitted to hospital; within two days he had a rolled mass across the epigastrium, free fluid in the abdomen and his tuberculin test had been shown to be positive. Streptomycin (I g. daily) and I.N.H. (200 mg. daily) were given with an almost immediate improvement in his general condition.

The family were then examined and his elder sister, aged six, was also found to be tuberculin positive and, as it so happened, she was known to have been negative a year earlier, when she was examined at school entry. Shortly after she started school the family had moved from the grandparents' home in another village six miles away, to their present house. The parents and the third child, an infant, seemed well and both father and mother had normal films on radiological examination. The infant had a negative tuberculin test and was breast fed.

I then remembered that in another hospital I had under my care an infant of 15 months of age who lived in the next street in the same village and was suffering from tuberculous glands of the neck.

We soon found that both families obtained their milk from the same neighbouring farm and the Medical Officer of Health found a tuberculous cow among the milking cattle. Whilst this investigation was in progress a fourth child was seen with tuberculous cervical glands.

All four children were treated and recovered without the development of any complications except a local gland abscess.

\section{Pathogenesis of Lymphadenitis}

There has been great speculation about the relationship of superficial lymphadenitis to the primary tuberculous infection; and I have recently reviewed my own material attempting to study this point. In a series of $16_{3}$ children with proven tuberculous adenitis there was evidence that in at least 80 per cent. and possibly as many as 90 per cent. the superficial glands, wherever they presented, were the regional glands of the primary complex of tuberculosis (Miller and Cashman, 1958). In a few of these children the peripheral, adenitis was associated with evidence of earlier tuberculosis either in the lungs or abdomen and the precise relationship of the two 


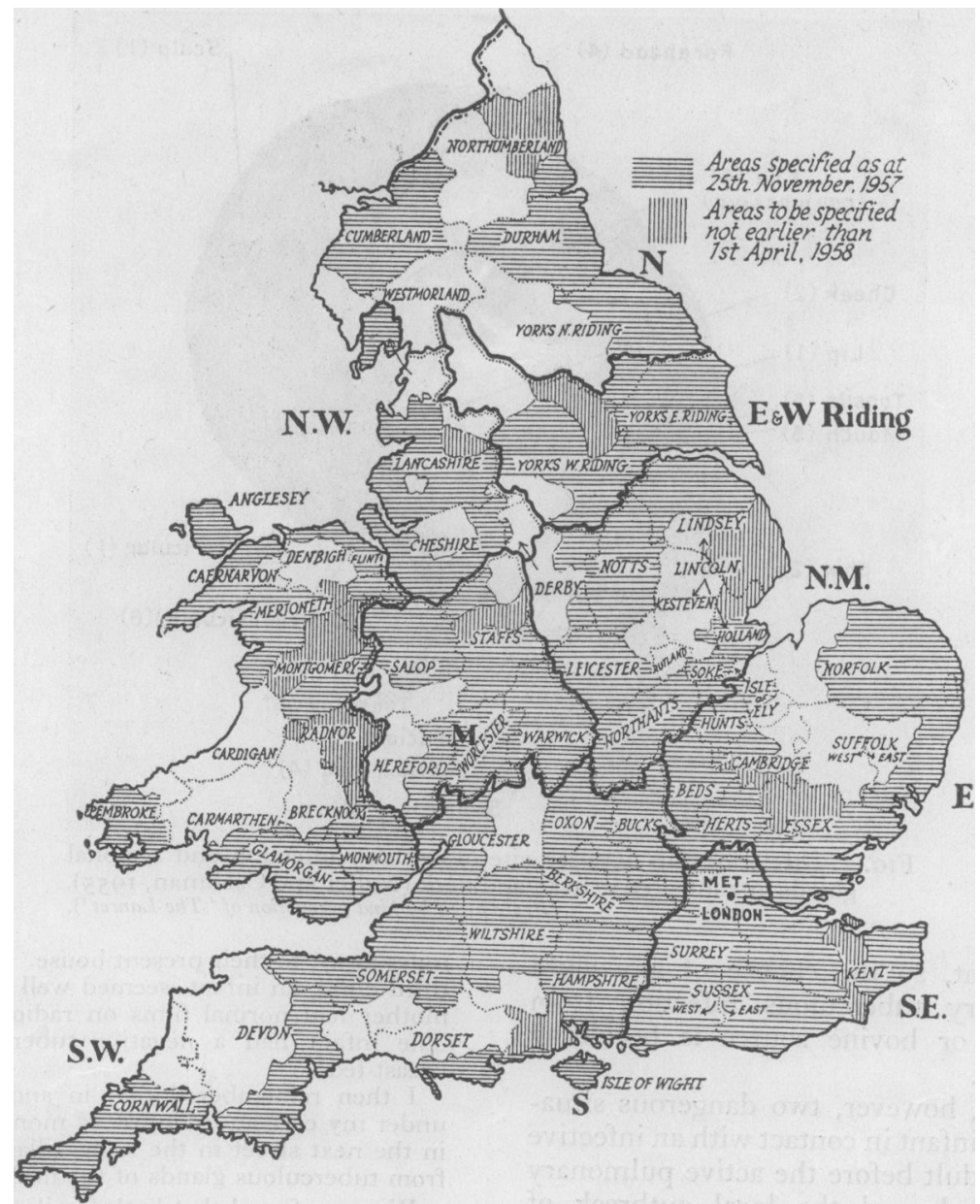

Fig. 2-Specified areas in which all milk sold by retail must be specially designated (that is, pasteurized, sterilized or tuberculin tested, The Medical Officer, 1958).

(Reproduced by kind permission of ' The Medical Officer').

manifestations of disease could not be determined. But this is uncommon and I believe that, in childhood, peripheral lymphadenitis is nearly always directly connected with the drainage of a primary focus.

\section{Natural History}

At the time of the primary infection a small tuberculous focus forms at the site of invasion and the regional glands enlarge to an extent which varies with the age of the child, the heaviness of the infection and the degree of sensitivity developed. The first gland infected undergoes the greatest enlargement, but those which drain from it are also infected, become enlarged to 'some degree and in this way a chain of infected glands is produced.

During this time, tubercle bacilli escape inter- mittently into the blood stream, and a small minority of children develop clinical illnesses in the form of localized haematogenous lesions or generalized disease if the infection is heavy and resistance low. This escape of organism occurs to some extent in all children undergoing primary infection and is the reason why primary tuberculosis should always be considered a general rather than a local disease.

The primary focus heals slowly but rarely gives rise to trouble. The regional glands remain enlarged and if they are not dealt with sooner or later in almost all cases of peripheral adenitis a local abscess is formed, usually at the site of the gland nearest the primary focus. Of the $16_{3}$ children quoted above, only in nine had the glands failed to soften by the time of the review. In a smaller series of 40 children with peripheral glands 
TABLE I

Relationship of Calcification to Time of Infection

(Miller and Cashman, 1955)

\begin{tabular}{|c|c|c|c|c|c|c|c|c|c|c|c|c|}
\hline \multirow{2}{*}{\multicolumn{3}{|c|}{$\begin{array}{l}\text { Radiographic Examination } \\
\text { of Regional Glands }\end{array}$}} & \multirow{2}{*}{ Calcification } & \multicolumn{8}{|c|}{ Years after Infection } & \multirow{2}{*}{ Total } \\
\hline & & & & 0 & I & 2 & 3 & 4 & 5 & 6 & 7 & \\
\hline \multirow{3}{*}{$\begin{array}{l}\text { First } \\
\text { Subsequent }\end{array}$} & & \multirow{3}{*}{$\begin{array}{l}\cdots \\
\cdots\end{array}$} & Present & - & 4 & I & 6 & I & $\circ$ & $\mathbf{I}$ & I & 14 \\
\hline & & & $\begin{array}{l}\text { Present for first time } \\
\text { Still absent }\end{array}$ & - & $\begin{array}{l}0 \\
6\end{array}$ & $\begin{array}{l}7 \\
7\end{array}$ & $\begin{array}{l}4 \\
6\end{array}$ & I & 2 & $\overline{1}$ & $\frac{1}{2}$ & 14 \\
\hline & & & Total calcified & - & 4 & 12 & 22 & 24 & 26 & 27 & 28 & 28 \\
\hline
\end{tabular}

associated with a visible primary infection all the glands not surgically removed when firm eventually formed abscesses.

Although some glands do not soften for years after infection, in most cases they do so within the first year; thus in the 40 children mentioned above, 25 had gland abscesses within six months and 29 within a year of infection. Furthermore, in children who are not given chemotherapy, if abscess formation occurs there is a significant risk of further abscesses either at the same site or in glands further down the lymphatic chain.

The glands also calcify, and in England the radiological identification of calcium is an indication both of the nature of the adenitis and the time of the original infection. Calcium is uncommon in the first year after infection and appears most commonly in the second or third year afterwards (Miller and Cashman, 1955).

As far as I can determine, the child with peripheral tuberculosis undergoes the same risks of miliary tuberculosis, tuberculous meningitis or haematogenous lesions at the time of primary infection as the child whose primary complex is in the lung or the abdomen. Unless, however, he is unfortunate enough to have a primary focus in the lung in addition to one on the mucous membrane or skin, he escapes the risk of tuberculous endobronchitis or the aspiration-segmental lesions which attend mediastinal lymphadenitis and has a lesser risk of developing pulmonary tuberculosis afterwards.

\section{Clinical Syndromes of Tuberculous Lymphadenitis}

Tuberculous peripheral adenitis presents in one of two situations; the first is immediately after or within a few months of infection. The glands may still be firm or they may already have softened. The second situation is when a calcified or partly calcified gland which has been infected for a considerable period of time breaks down and softens. This not uncommonly follows a non-specific respiratory infection, tonsillitis or one of the acute specific fevers of childhood and appears to be due to an altered state of ' resistance ' of the host.

\section{Recently Infected Glands: Usual Onset}

The commonest clinical syndrome is when the child is brought to the doctor with a painless enlargement of a lymphatic gland. This enlargement has usually been noticed accidently as the child turned his head or found as he was being washed. As a rule he is thought to be in his ordinary health but close observation and questioning will almost always reveal that this is not so, for he is less active than formerly, looks pale and is likely to have lost his appetite. He may or may not have a slight evening temperature.

On examination the gland is painless, and is not attached to skin or the deeper tissues unless it is softening when the overlying skin tissue becomes pink and then red. Other glands in the course of the lymphatic drainage are always enlarged but their size diminishes with the distance from the main enlargement.

If the gland remains untreated at this stage it is likely to soften within a few weeks or months; sometimes the swelling progresses to softening without any remission, at other times it reaches a maximal size then regresses for a while, only to enlarge again and then proceed to softening. Occasionally this process may be repeated more than once before the abscess forms.

Less often the regression continues and the patient and his parents may consider that the gland has disappeared and therefore all is well. It does not, however, return completely to its original size before infection but remains palpable and firm and slowly, after a year or more, shows evidence of healing by the appearance of calcification.

The usual onset is, therefore, slow and undramatic and two of the most noticeable features, in sharp contrast to the adenitis of an acute pyococcal infection, are the lack of pain and the absence of any muscle spasm or loss of function in the affected part of the body. 


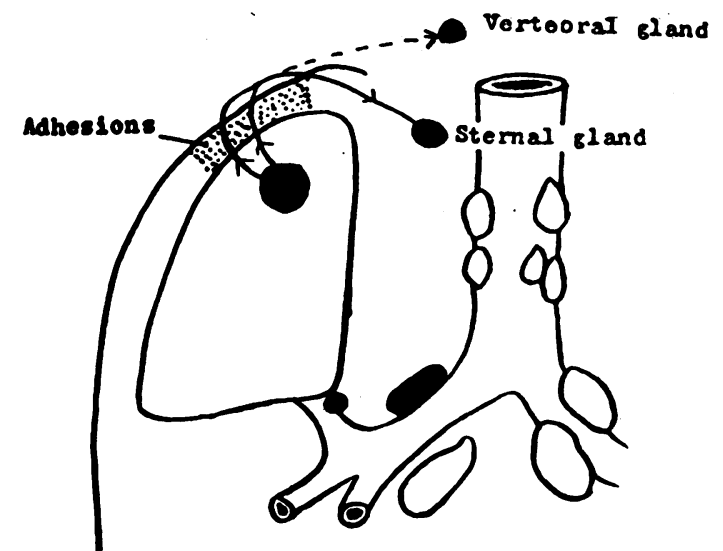

FIG. 3.-Drainage of apical primary focus to the sternal and vertebral glands in addition to the mediastinal glands (Scott and Beattie, 1928).

\section{Recent Infection: Acute Form}

But not all tuberculous infections have this slow onset. Occasionally, in a child who has either a heavy infection or develops a marked sensitivity, the reaction is much more acute in both its local and general forms. The swelling of the gland comes in a few days and at the same time the child becomes ill and toxic while his temperature ranges from $\mathrm{IOI}^{\circ}$ to $\mathrm{IO}^{\circ}$. Although swollen and rather tense, the glands are not acutely painful, although if the child is apprehensive that impression can be obtained.

The sharpness of the reaction usually makes the initial diagnosis one of acute non-tuberculous adenitis and sometimes the real cause is not suspected until an abscess has formed and the pus is examined. Glands which enlarge in this way coalesce to form a large mass and soften faster than those with a less dramatic onset.

\section{Glands which Break Down after Initial Healing}

Although this is most likely to occur in the first gland affected it can occur anywhere on the lymphatic drainage and might account for the appearance of a tuberculous adenitis in an unusual situation, e.g. the supraclavicular glands (Fig. 3).

This child also presents with a painless gland swelling which has almost always involved the skin and may, indeed, have ruptured spontaneously. The onset of the swelling and softening is only a matter of two to four weeks, and there may or may not be a history of previous swelling of the glands or the knowledge that for some considerable time the gland, which has recently swollen, had been $z$ hard and easily palpable. On radiological $\stackrel{\mathbb{Q}}{\complement}$ examination calcification is often seen.

\section{Diagnosis of Tuberculous Lymphadenitis}

When the glands are firm and only moderately enlarged, the diagnosis cannot be made with cer- $\frac{\bar{\sigma}}{\bar{\omega}}$ tainty on clinical grounds alone. The major $\vec{\nabla}$ difficulties occur in tonsillar adenitis, when the $\propto$ distinction must be made between an early tuberculous infection in a child who is not very ill and a $\overrightarrow{0}$ chronic non-specific enlargement which may accompany an upper respiratory infection. The $\vec{\omega}$ latter is much more usually bilateral than the former, but on clinical examination the glands may feel alike in all respects. For even the adenitis $\frac{3}{\dot{\omega}}$ which accompanies an acute non-specific ton- ov sillitis is not always tender to touch.

In tuberculous lymphadenitis the skin tuberculin test will always be positive and, if the infection is recent, there may also be sensitivity phenomena such as erythema nodosum or phlyctenular conjunctivitis. The spleen is often enlarged during $\vec{c}$ the initial period of primary tuberculous infection but it can also be felt after an attack of streptococcal tonsillitis. X-ray of the chest or abdomen and of $\vec{v}$ the affected region may reveal calcification of other signs of local or haematogenous tuberculosis. When the presenting glands are calcified, any re? cent enlargement is almost certainly due to reactivity of the tuberculosis. If a local abscess has already formed, the pus should be examined. If the abscess is tuberculous, a few organisms may be seen in a direct film but the pus is more likely to be sterile. If the glands are firm, the tuberculin test is positive and the clinical evidence suggests that the enlargement is due to tuberculosis, the next stage is a therapeutic trial.

\section{Treatment of Tuberculous Lymphadenitis}

The treatment of tuberculous lymphadenitis must be designed to make the period of illness as short as possible, to obtain a good cosmetic result 을 and to prevent the development of complications remote in time or situation. The cosmetic result depends largely upon the control of abscess formation and the care with which any surgical procedure $\sigma$ can be planned and executed. If we accept that, $N$ if untreated, nearly all peripheral tuberculous lym- N phadenitis will form abscesses, then two questions $\sigma$ remain: can this abscess formation be prevented by chemotherapy given in adequate doses and con- $\frac{\widetilde{C}}{\Phi}$ tinued for a sufficiently long time? Can subsequent $\stackrel{P}{?}$ abscess formation be prevented if firm glands are 0 removed by local dissection whilst the child is treated with chemotherapy?

In my experience chemotherapy alone is not 
sufficient to prevent softening of glands, even when it is continued for more than a year. Originally streptomycin and P.A.S. were used, and later I.N.H. and P.A.S. The latter combination can be given for long periods without difficulty. The effect of giving chemotherapy to children with firm glands recently infected is interesting, there is an immediate response which is proportional to the original size of the glands. Thus in a febrile child with an acute reaction, and a large mass of glands with much peridenitis, the temperature falls rapidly, and the swelling shrinks, often resolving into two or more discrete glands. In a child with a small gland and little change in general health or bodily reaction the change is correspondingly less. But all children improve in health and well-being and all recently infected glands show some reduction in size when first treated. This improvement continues for about three to four weeks and then stops, leaving a firm, movable, painless gland. If the chemotherapy is continued, the gland may remain in this state for many months but those we have observed have softened ultimately. Once softening occurs, skin involvement is inevitable and then the chance of achieving a good cosmetic result has largely gone.

In looking after a child with recent tuberculous infection of glands I therefore use chemotherapy and continue as long as necessary until the first sign of secondary enlargement of the gland occurs. Then I advise removal of the whole gland and its immediate neighbours by careful local dissection. No attempt is made to remove all infected glands, for infection has spread far down the lymphatic chain, but great care is taken with the final suturing of the wound. Chemotherapy is continued for at least a year after the infection. The prolonged chemotherapy has two purposes: to help to avoid further local abscesses and to prevent haematogenous lesions in other organs. Chemotherapy is just as necessary when the lymph glands are superficial and visible as it is when the infection is pulmonary or mesenteric.

Streptomycin ( $45 \mathrm{mg}$. per kilo, $20 \mathrm{mg}$. per I lb. body weight, with a maximum dose of $\mathrm{I}$ g. per day) and I.N.H. (20 mg. per kilo, maximum $400 \mathrm{mg}$.) are used to cover the operation and until the wound is firmly healed. The daily injection of streptomycin is then stopped and P.A.S. (125 mg. per I lb., $300 \mathrm{mg}$. per kilo) is given instead and continued for at least a year.

If the glands are soft when first seen, the situation is not so favourable, but, whether infection is recent or old, the general problem is the same and chemotherapy should be given as described above. When there is calcium present and infection has been present for a considerable time there is not the same risk of new haematogenous spread but I.N.H. and P.A.S. should be given for six months.

The basic trouble when the gland has softened is that it cannot be removed as a whole, and if caseous material is left at the site of the abscess then there is always a risk of subsequent abscess formation. Unless the contents of the gland are exceptionally fluid, mere incision is not likely to remove all caseous material; for this reason other methods, such as curettage or expression, have been used, and more recently Lincoln (1957) has used enzyme irrigation to achieve the same purpose. If the caseous material can be removed healing will follow, but the final appearance of the scar depends upon the amount of skin loss.

The early recognition and effective treatment o tuberculous lymphadenitis in this way can reduce the length of illness, the chance of complications and avoid the pigmented scars seen a generation ago.

In conclusion, therefore, it can be seen that the situation with regard to peripheral tuberculous lymphadenitis is changing rapidly, new cases are becoming less and less common as the sources of infection are brought under control, but old cases infected some time ago continue to present for treatment. Peripheral tuberculous lymphadenitis is also still to be seen in adults but, as I indicated at the beginning of this paper, its natural history seems different from that of tuberculous adenitis in childhood and it may require different treatment.

\section{BIBLIOGRAPHY}

ANASTASIADES, A. A., TSIKOUDAS, E. C., LINCOLN,

E. M., and DALY, J. F. (1957), Amer. Rev. Tuberc., 76, 588 . MILLER, F. J. W., and CASHMAN, J. M. (1955), Lancet, i, 1286. MILLER, F. J. W., and CASHMAN, J M. (1958), Ibid.,

MILLER, F. J. W. (I953), Ibid., i, 3.

SMITH, C. M. (1958), Med. Offr., 99, 45.

\section{Erratum May Issue}

We apologize for the illustration appearing in Dr. J. D. H. Slater's article on 'Oral Hypoglycaemic Drugs' which we regret was included in error. 